\title{
The AGATA Physics Campaign In GANIL: Results And Perspectives
}

\author{
Silvia Lenzi* \\ Padova University and INFN \\ E-mail: Lenziepd.infn.it

\section{Emmanuel Clément} \\ Affiliation GANIL, CEA/DRF-CNRS/IN2P3, Bd Henri Becquerel, BP 55027, F-14076 Caen, \\ France \\ E-mail: emmanuel.clementeganil.fr \\ Andrés Gadea \\ Affiliation Instituto de Física Corpuscular, CSIC-Universidad de Valencia, E-46071 Valencia, \\ Spain \\ E-mail: gadeadific.uv.es
}

AGATA is a $\gamma$-ray spectrometer solely built from Germanium detectors, based on the novel technique of $\gamma$-ray tracking. This array opens unique possibilities for a very rich physics program to be addressed, in particular on exotic nuclei where different nuclear degrees of freedom can be investigated. The AGATA physics campaign in GANIL started in 2015 and foresees different setups to study nuclear structure properties of nuclei all along the nuclear chart, from light nuclei to very heavy species, using stable and radioactive beams.

The 26th International Nuclear Physics Conference

11-16 September, 2016

Adelaide, Australia

${ }^{*}$ Speaker. 


\section{Introduction}

The Advanced Gamma Tracking Array, AGATA [U], is a new-generation high-resolution $\gamma$ ray spectrometer, based on the novel technique of $\gamma$-ray tracking. Since several years, the European $\gamma$-spectroscopy research community is involved in the construction of this high-efficiency Ge-detector array which has started its physics campaign in 2010 in LNL (Italy), and continued in GSI (Germany), in configurations that included a small fraction of the 180 detectors planned for the complete array. In 2014 AGATA was installed in GANIL (France), for a new physics campaign with an increased number of detectors.

GANIL offers the possibility of having high-intensity beams of stable nuclei up to Uranium as well as radioactive beams from SPIRAL1. This combination allows to study a large variety of nuclear properties all along the nuclear chart with $\gamma$-ray spectroscopy techniques, coupling AGATA to different complementary instrumentation.

The physics campaign in GANIL started in 2015 and has been organized in a bottom-up approach, following calls of letters of intent that have been discussed yearly in dedicated AGATA workshops. Among the proposed physics cases to be studied in the GANIL campaign we can mention: the evolution of the shell structure in neutron rich nuclei and the effect of three-body forces, exotic nuclear shapes and shape-coexistence phenomena, proton-neutron pairing and isospin symmetry along the $N=Z$ line, clusterization phenomena, the effect of the coupling to the continuum in weakly-bound systems, the structure of superheavy nuclei, structure properties of nuclear astrophysics interest, etc.

After a brief description of the AGATA concept, some recent results will be discussed together with the very interesting opportunities for nuclear structure research in the forthcoming years with AGATA at GANIL.

\section{The AGATA array at GANIL}

The AGATA citeAGATA array is based on the use of highly segmented HPGe crystals grouped in cluster in a single cryostat. Each AGATA crystal provides 38 signals: 36 for the segments and two for the core, namely two gains corresponding to about $5-\mathrm{MeV}$ and $20-\mathrm{MeV}$ full range. The output of the respective preamplifier is digitized by means of a 100-MHz 14-bit FADC. This information is sent via optical links to pre-processing cards, which perform the task of extracting the energy and time of a particular detector element. To access the energy and time information, the Moving-Window Deconvolution (MWD) technique and a digital constant fraction threshold algorithm are used, respectively. The outputs at this stage are transmitted to a computer farm performing further data processing like the on-line Pulse Shape Analysis (PSA), event-building and Tracking Analysis (TA).

The AGATA array installed at GANIL consists of triple (TC) and double (DC) clusters, organized in a compact geometry of up to 45 detectors to optimize the performance of the tracking array. The AGATA clusters are grouped at the most backward angles, symmetrically around the optical axis of the VAMOS++ spectrometer [0]. This arrangement maximizes the peak-to-total ratio, the Doppler effect correction and the efficiency for lifetime measurements. Indeed, thanks to the performances of both the PSA and the TA, consisting in a major improvement with respect 
to the EXOGAM array, the whole solid angle covered by the AGATA clusters can be used for lifetime measurements based on the analysis of Doppler shifts. With respect to the LNL and GSI campaigns, the number of clusters was increased from 5 (7) clusters in the LNL (GSI) campaign respectively, to $8 \mathrm{TC}$ in 2015 and $10 \mathrm{TC}+1 \mathrm{DC}$ in 2016 at GANIL. In 2017, for the third run, the AGATA system will consist of $11 \mathrm{TC}$ and $1 \mathrm{DC}$ with an expected photopeak efficiency greater than $10 \%$ for a $1.3 \mathrm{MeV} \gamma$-ray at a detectors distance of $13 \mathrm{~cm}$. This increase of the number of channels has led to the production of additional modules of the Detectors Support System and the development of a second generation of Front-End and Back-End preprocessing electronics.

The AGATA campaign exploits the stable and radioactive heavy ions beams delivered by the cyclotron accelerator complex of GANIL. Additionally, it benefits from a large palette of complementary detectors and spectrometers to address in-beam $\gamma$-ray spectroscopy of exotic nuclei. The set-up has been designed to couple AGATA with a magnetic spectrometer, charged-particle and neutron detectors, scintillators for the detection of high-energy $\gamma$ rays and other devices such as a plunger to measure nuclear lifetimes. In 2015-2016 AGATA was coupled to the VAMOS++ spectrometer that identifies the exotic nuclei produced in heavy-ions collisions at the Coulomb barrier. Nuclear lifetimes were measured using both the plunger and the DSAM techniques. In 2017, scintillator detectors from the FATIMA [B] and PARIS [䧃] collaborations will be added to the setup to further increase its sensitivity. The high energy $\gamma$-ray efficiency will be improved and fast-timing methods will be used to probe nuclear lifetime in the nanoseconds range.

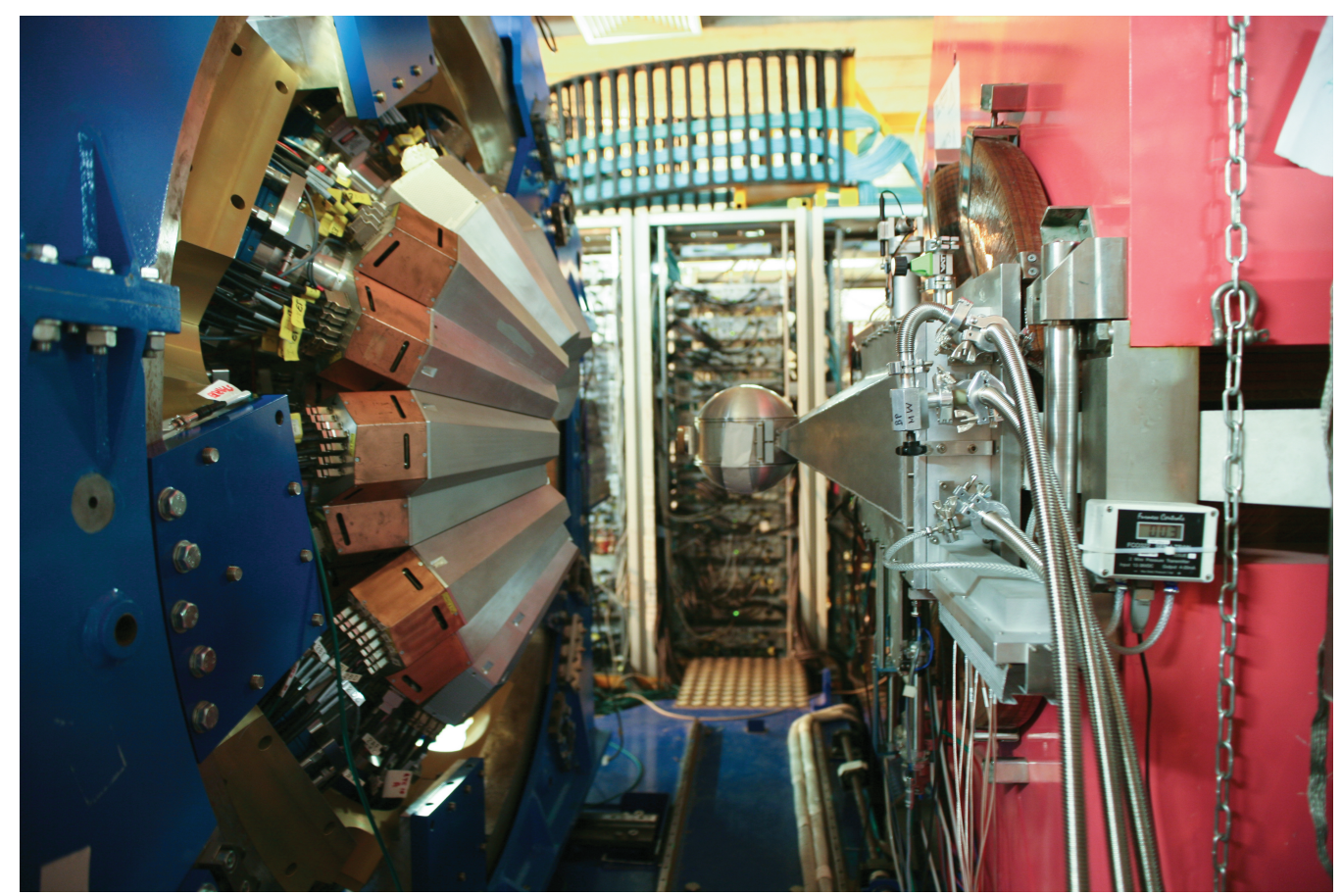

Figure 1: The setup shows AGATA coupled to the magnetic spectrometer VAMOS++ 


\section{First Campaign: AGATA coupled to VAMOS++}

Four different setups have been identified since the first collaboration workshop held in GANIL for the AGATA campaign in 2013. The first setup, consisting on AGATA coupled to the largeacceptance magnetic spectrometer VAMOS++ []], started taking data in 2015. The main reaction mechanisms used to populate excited states in nuclei far from stability are multi-nucleon transfer and fusion-fission. Some experiments aimed at measuring lifetimes by means of the Recoil Doppler Distance Shift method for which plunger devices were placed at the target position [ [1, 目]. Between the 2015 and 2016 runs, the beamtime devoted to AGATA experiments has been of about 100 days.

A wide physics program is covered by the performed experiments that aimed at investigating single-particle and collective degrees of freedom in nuclei far from the valley of stability. Nuclear structure properties such as shell evolution and the development of deformation near magic numbers, exotic shapes and shape coexistence phenomena, lifetimes measurements of astrophysics interest have been investigated. The run of 2017 will see, coupled to AGATA, large arrays of scintillator detectors from the PARIS and FATIMA collaborations for the study of subjects related to the effect of three-body forces in light nuclei, shape transitions in rear earth nuclei, shape coexistence in fission fragments and alpha-clusterization in heavy nuclei.

The study of the evolution of the shell structure far from stability and the development of Islands of Inversion are current research goals at different accelerators of both stable and radioactive beams. This is a complex problem that needs to be approached by means of the measurement of different observables. The first performed experiment with AGATA at GANIL aimed at measuring lifetimes of excited states in $\mathrm{Co}, \mathrm{Fe}$ and $\mathrm{Mn}$ isotopes at the Island of Inversion south of ${ }^{68} \mathrm{Ni}$, populated via the multi-nucleon transfer ${ }^{238} \mathrm{U}+{ }^{64} \mathrm{Ni}$ reaction in inverse kinematics, using the OUPS plunger [可]. Lifetimes of the $4^{+}$states in ${ }^{62,64} \mathrm{Fe}$ and the $11 / 2^{-}$states in ${ }^{61,63} \mathrm{Co}$ and ${ }^{59} \mathrm{Mn}$ were measured. The extracted experimental $B(E 2)$ values have been compared with large-scale shell model calculations using the LNPS interaction [ [D] and with beyond mean-field calculations using the Gogny D1S interaction [ [ ] ] An increase of quadrupole collectivity approaching $N=40$ is observed in all isotopes. This work has been recently accepted for publication [Q]. Lifetimes in $\mathrm{Ti}$ isotopes that lie at the border of this Island of Inversion have also been measured in another experiment. The analysis of these data is in progress as well as those of obtained recently for the lifetime measurement of neutron-rich $\mathrm{Ga}$ and $\mathrm{Zn}$ isotopes aiming at mapping collectivity changes towards $N=50$.

The experimental method of populating proton-rich nuclei by multi-nucleon transfer reactions has been used by two experiments with AGATA. The first proposed to investigate the first excited states in ${ }^{106,108} \mathrm{Sn}$ populated using a ${ }^{106} \mathrm{Cd}$ beam on a ${ }^{58} \mathrm{Ni}$ target. Previous measurements of the $B\left(E 2 ; 2^{+} \rightarrow 0^{+}\right)$in these isotopes present large error bars and depart from the parabolic behaviour expected from shell model calculations in the truncated $g d s$ space. This unconventional reaction mechanism for studying proton rich nuclei directly populates low spin states with large probability bypassing the isomeric $6_{1}^{+}$state, thus allowing the measurement of the lifetime of the $2^{+}$states with the differential plunger method. The second experiment using this experimental method aimed to measure the lifetimes in even-even $N=50$ isotones. These nuclei present similarities with $\mathrm{Ni}$ isotopes, in the sense that neutrons in $\mathrm{Ni}$ isotopes are filling the same orbitals than protons in $N=50$ isotones. Indeed, the energy of the lowest $2^{+}$and $4^{+}$states are quite similar for ${ }^{70-76} \mathrm{Ni}$ 
isotopes to those in ${ }^{92} \mathrm{Mo},{ }^{94} \mathrm{Ru},{ }^{96} \mathrm{Pd}$ and ${ }^{98} \mathrm{Cd}$. For the transition probabilities $B\left(E 2 ; 2^{+} \rightarrow 0^{+}\right)$ shell model calculations by Lisetskiy et al. [ए]] in the fpg space predict a similar trend for the $N=50$ isotones and $Z=28$ isotopes, while a relative inversion of the trend is predicted for the $B\left(E 2 ; 4^{+} \rightarrow 2^{+}\right)$values, probably owing to the strong pairing predicted for the $N=50$ isotones near ${ }^{100} \mathrm{Sn}$. Very scarce information was available in this mass region before this experiment that will give a final answer to this puzzle.

There is currently a discussion on the size and stability of the gap at the magic number $N=50$ in very neutron-rich systems, together with the energy of the single-particle levels around it. To investigate this mass region the ideal reaction mechanism that exploits the optimum characteristics of the AGATA plus VAMOS++ setup is the fusion-fission reaction in inverse kinematics. Exotic species can be selected by properly choosing the angle of VAMOS++ with respect to the beamline. Two experiments have been performed with this technique. The first one aimed at the measurement of the lowest $6^{+}$and $7^{+}$excited states in ${ }^{80} \mathrm{Zn}$ and ${ }^{82} \mathrm{Ge}$. These states, that involve a particle-hole excitation across the $N=50$ gap, are predicted to follow the same trend as that of the gap, as a function of the proton number [ए], [2]. A local minimum in the effective $N=50$ gap, defined as $\Delta=S_{2 n}(N=52)-S_{2 n}(N=50)$, has been found at $Z=32$ and an increase is predicted going from $Z=32$ to $Z=30$. This would allow to probe the robustness of the $Z=28$ gap near ${ }^{78} \mathrm{Ni}$. The analysis of these data is in progress. Meanwhile, other isotopes produced in this experiment are being studied. In particular, new excited states have been evidenced in ${ }^{96} \mathrm{Kr}$ that show a sudden transition from strongly deformed to almost spherical shapes in the $N=60$ isotones. A publication on these results is in preparation [103].

The evolution of the effective single-particle energies above $N=50$ is still an open question and recent experimental findings have put in evidence unpredictable behaviour. Low-lying states in $N=51$ isotones can be interpreted in terms of a single-particle configuration or as the coupling of the single particle to a core excitation. The latter being of collective character. To disentangle the character of a state, the measurement of the lifetime constitutes a good choice as the collective state will have a much shorter lifetime that that of a single-particle configuration. For this purpose an experiment has been performed using a fusion-fission reaction and the plunger device coupled to AGATA and VAMOS++. Data on ${ }^{83} \mathrm{Ge}(Z=32)$ are being analyzed. Apart from the above described experiments, other series of experiments have been performed successfully and are under analysis.

\section{Future Campaigns}

For the 2018 run AGATA will be coupled to the NEDA/Neutron-Wall array [4], प5] and the charged-particle detector DIAMANT [ [6] for a very ambitious scientific program to study neutrondeficient nuclei and the structure of $N \sim Z$ nuclei.

The approved experimental program at GANIL aim at studying proton-neutron pairing effects, isospin symmetry breaking in mirror nuclei, shape-coexistence phenomena at $N=Z$ near $A=70$ via lifetime measurements, shell structure near ${ }^{100} \mathrm{Sn}$ together with octupole correlations, clusterization phenomena in light nuclei and superdeformation. The final mechanical design of NEDA + Neutron-WALL foresees the use of 54 self-produced NEDA detectors at forward angles and 14 Neutron-WALL detectors at around 90 degrees, as can be seen in Fig. 2. 


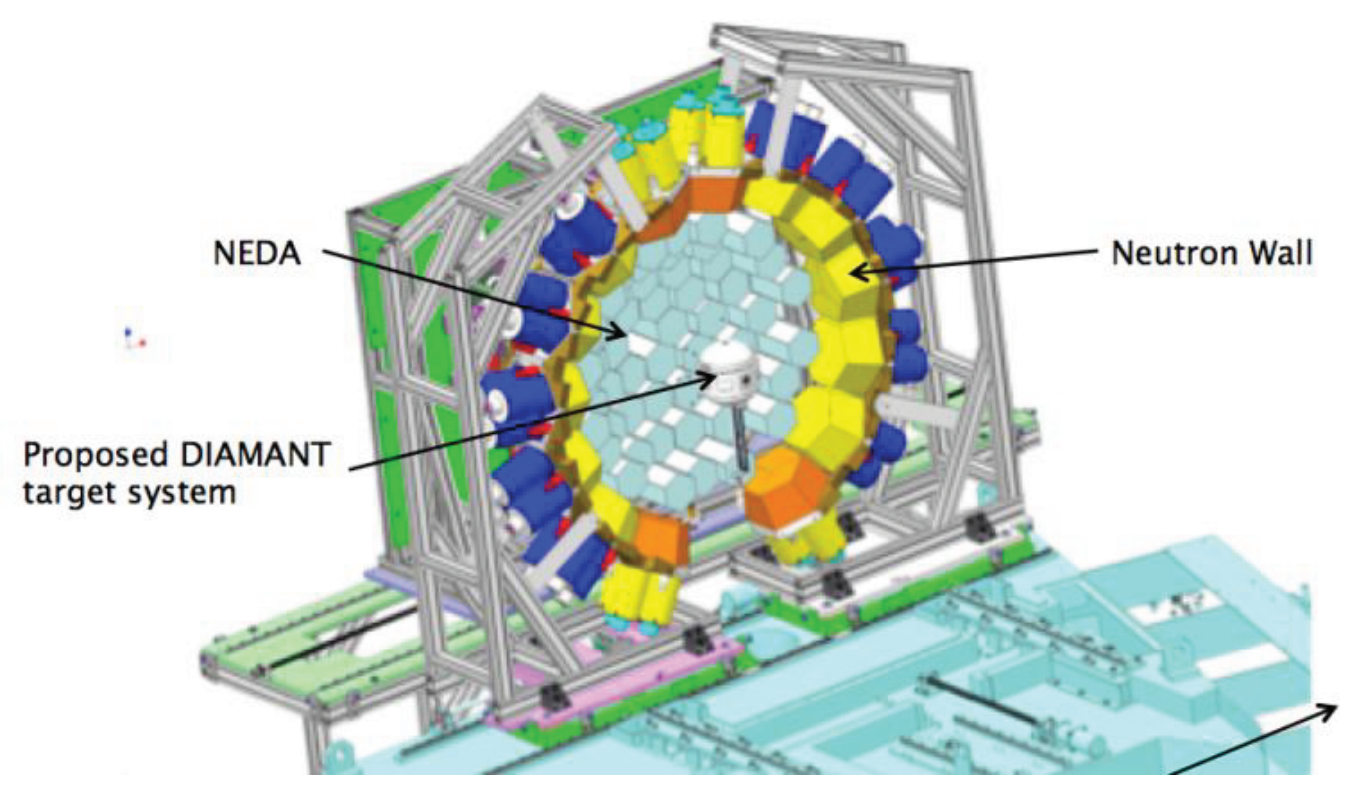

Figure 2: The setup shows AGATA coupled to NEDA, Neutron-Wall and DIAMANT

Two letters of intent have been submitted to the GANIL PAC proposing two different detectors to be coupled to AGATA: MUGAST, that will use radioactive beams from SPIRAL1, and VAMOS in gas-filled mode[[7], [8]. Both setups are in construction and will be ready to take data since 2019. Several proposals have been presented and discussed at the AGATA Workshop organized in GANIL in February 2016: 10 of them for VAMOS in gas-filled mode and 16 requesting the SPIRAL1 beams, showing a great interest of the users community in the AGATA campaign at GANIL.

\section{Conclusions and Perspectives}

The AGATA physics campaign in GANIL, started in 2015 has attracted the interest of a large community for the study of nuclear structure and reactions dynamics of nuclei far from the valley of stability by means of gamma spectroscopy. The high intensity stable beams delivered by the GANIL cyclotrons up to U, together with the radioactive beams of SPIRAL1 allow to use different reaction mechanisms to populate the nuclei of interest. The sensitivity of AGATA largely increases by the coupling to particle detectors. In particular, the first campaign of experiments using the magnetic spectrometer VAMOS++ and, in some cases the plunger device, has produced quite successful results. For 2017 the setup will be further improved by the coupling of several scintillator detectors from the FATIMA and PARIS collaboration. The following setup will see AGATA coupled to NEDA/Neutron Wall and the light charged-particle detector array DIAMANT in 2018. Afterwords two different setups are foreseen. VAMOS in gas-filled mode will be coupled to AGATA for the study of heavy $N=Z$ and super heavy elements. The charged-particle array MUGAST will be used with radioactive beams with the aim of studying properties of astrophysics interest, shell evolution, clusterization phenomena and other interesting subjects. 


\section{References}

[1] S. Akkoyun et al., Nucl. Instr. and Meth. A 668 (2012) 26.

[2] M. Rejmund et al., Nucl. Instr. and Meth. A 646 (2011) 184.

[3] J.-M. Rgis et al., Nucl. Instr. and Meth. A 622 (2010) 83.

[4] A. Maj et al., Acta Physica Polonica B 40 (2009) 565.

[5] J. Ljungvallet al., Nucl. Instr. and Meth. A 67961 (2012).

[6] A. Dewald et al. Nucl. Instr. and Meth. A 679 (2012) 61.

[7] S. M. Lenzi, F. Nowacki, A. Poves, and K. Sieja, Phys. Rev. C 82 (2010) 054301.

[8] L. Gaudefroy, A. Obertelli, S. PÃl'ru, N. Pillet, S. Hilaire, J. P. Delaroche, M. Girod, and J. Libert, Phys. Rev. C 80 (2009) 064313

[9] M. Klintefjord et al., Phys. Rev. C, in press (2017).

[10] A.F. Lisetskiy et al., Eur. Jour. Phys. A 25 (2005) S01.

[11] M. Bender et al., Phys. Rev. C 78 (2008) 054312.

[12] K. Sieja and F. Nowacki, Phys. Rev. C 85 (2012) 051301(R).

[13] J. Dudouet et al., to be submitted.

[14] Tayfun Hüyük et al., Eur. Phys. J. A 52 (2016) 55; J.J. Valiente-Dobón et al., in preparation (2017).

[15] Ö. Skeppstedt et al., Nucl. Instr. and Meth. A 421 (1999) 531

[16] J. Scheurer et al., Nucl. Instr. and Meth. A 385 (1997) 501.

[17] C. Schmitt et al., Nucl. Instr. and Meth. A 621 (2010) 558.

[18] C. Theisen et al., Nucl. Instr. and Meth. A 747 (2014) 69. 